\title{
Exploring the Importance of Modification Relation for Emotional Keywords Annotation and Emotion Types Recognition
}

\author{
Yunong Wu*, Kenji Kita, Fuji Ren, Kazuyuki Matsumoto, Xin Kang \\ Faculty of Engineering, University of Tokushima, Tokushima, Japan \\ * Corresponding author's Email: wuyunong@iss.tokushima-u.ac.jp
}

\begin{abstract}
In this study, we make a scheme to explore the importance of modification relation for the emotional keywords annotation and emotion types recognition. We extract three modification features which are degree words, negative words and conjunctions from the Chinese emotion corpus named Ren-CECps. Beside word and part-ofspeech, three modification relations are adopted as feature in this study. We have carried out eight experiments with different feature sets for emotional keywords annotation and emotion types recognition in sentence level. Eight basic emotion types have been selected and Conditional Random Fields have been employed as the algorithm. In the part of evaluation, we demonstrate the importance of the modification features and our experiment results show the effectiveness of the modification features for improving the performance of emotional keywords annotation and emotion types recognition.
\end{abstract}

Keywords: Emotional keywords annotation; Emotion types recognition; Modification relation; CRFs

\section{Introduction}

Affective information processing becomwii more and more important, recent emotion analysis mainly concentrates on the identification of people's inner sentiments. In general, emotion analysis can be divided into coarse classification and fine-grained classification. In the former study, researchers classify emotion into two classes including positive and negative or into three classes, in which neutral is added as a new class. While in the latter study, many significant emotion types are chosen for the emotion analysis, such as Expect, Joy, Love, Surprise, Anxiety, Sorrow, Anger and Hate.

Many researches have been worked on emotion classification using emotion lexicon based machine learning methods in textual analysis $[1,2]$. However the emotions of words in lexicon are static while in realworld they could be flexible, so experiments based on emotion lexicon may suffer from insufficient or mis- leading emotion features. And the recent work [3] shows that more precise word emotions improve emotion analysis of sentences.

In this paper we are focusing on emotional keywords annotation and emotion types recognition in sentence level. Since both emotional words and emotional phrases are the basic elements of the document, we regard them as emotional keywords in this study. The word emotions are not able to be recognized exactly by only utilizing the emotion lexicon, so we decide to explore the modification relations between words and the modifiers with respect to the sentences which involve the degree words, negative words and conjunctions, that would be helpful in acquiring more contextual information.

Except No_emotion, there are totally eight emotion categories in our scheme, namely, Expect, Joy, Love, Surprise, Anxiety, Sorrow, Anger and Hate. We use Ren-CECps ${ }^{1}$ Ren-CECps is a Chinese emotion corpus composed of 1,487 webblog articles, with 11,255 
paragraphs, 35,096 sentences, and 878,164 Chinese words. Those articles are manually annotated with detailed linguistic expressions and emotion tags. [4] as our data source which is manually annotated with both emotion categories and emotion intensities to different level of texts including words, sentences, phrases and documents. Degree words, negative words and conjunctions are also given the corresponding tags respectively as well.

Emotional word analysis in sentence is always accompanied with contextual emotion analysis. Traditional machine learning methods based on lexicon features in emotion classification and emotion tagging suffer low precisions for text components (words and sentences) in complicated language situations [5]. The example is shown as follows:

Sentence:“当经理看见小李在上班的时候看报 纸, 说: “你真会享受生活”。(When manager saw Lee reading newspaper at work, he said:"you really enjoy yourself.)"

In the example above, the word "enjoy" which is modified by the degree word "really", indicates a negative emotion of "critical", while this word conveys only a positive emotion of "take pleasure in something" in the lexicon. Inaccuracy could be caused misleading due to this emotion information from emotion lexicons. Because the emotions of words in emotion lexicon are static, when emotion analysis, which is conducted merely based on emotion lexicon in a complex context, would cause ambiguity of word emotions. However, our research explore the modifications relations between words and conjunctions, words and negative words, words and degree words respectively, would be helpful for conquering this problem.

We carry out eight experiments by using different features sets to explore the effectiveness of modification relations for emotional keywords annotation and emotion types recognition. In order to take contextual information into account, we regard Conditional Random Fields (CRFs) [6] as the appropriate algorithm for capturing the relevance between words and their corresponding modifiers in the same sentence.

The rest of this paper is organized as follows: Section 2 describes the related work. Section 3 introduces the data source. Experiment is demonstrated in detail in Section 4. Finally Section 5 concludes this paper and gives the future work.

\section{Related works}

Affective information processing becomes more and more important [21], especially emotion analysis got a lot of attentions from researchers. Many researches of emotion analysis have been focusing on sentiment polarities classification or some specific fine-grained emotions on three aspects: reviews [7, 8], feedbacks [9] and blogs [10, 11].

Most sentiment classification experiments were carried out by using Support Vector Machine (SVM) [12], Naive Bayes [18], Decision Trees [19], Maximum Entropy [20] and Conditional Random Fields (CRFs) [14]. The sentiment classification experiment implemented by [15] proved that CRFs classifiers outperform SVM classifiers.

[16] made an experiment, by using CRFs to tag emotions from words to sentences of Bengali. In his study, emotion tags are pre-assigned based on a Bengali SentiWordNet translated from English SentiWordNet. However, since the tagging procedure was based on an immovable word list, their result is bound to suffer from the curse of emotion lexicon with either high coverage/low precision or low coverage/high precision [5]. Contextual information was not considered in their work either.

To solve this problem, we explore Ren-CECps, a well developed Chinese emotion corpus with emotional words as well as modification components tagged out considering the contexts by human for emotional keywords annotation emotion types recognition.

We extract three modification relations as feature sets for acquiring more contextual information, and employ Conditional Random Fields as our algorithm for capturing the relevance between words and their modifiers.

\subsection{Emotion elements}

In our experiments, we make use of basic feature sets and modification feature sets for emotional keywords annotation and corresponding emotion types recognition. We view emotional keywords, which is composed of emotional words and emotional phrases, and part-of-speech tags as basic feature sets. Degree words, negative words and conjunctions constitute the modification feature sets. We introduce these emotion elements in detail as follows:

\subsubsection{Emotional Words}

Some words can always express the emotions directly. However, besides words directly referring to emotional states, several words indicate emotions indirectly depending on the context [17]. For examples:

Sentence 1: “我希望嫣儿长大了可以成为这样 的人。(I hope Yan could be such a person when she 
grows up.)"

Sentence 2: “冬天来了, 春天还会远吗? (If winter comes, can spring be far behind?)",

In the sentence 2, the word "spring" doesn't convey any emotion in usual, but in this context, it indicates the emotion of expectation.

Many emotional words are annotated with multiple emotions in the corpus. We choose the emotional type, corresponding the highest emotion intensity, as the chief emotion of the word. The numbers of emotional words in each category are shown in Table 1.

Table 1 The numbers of emotional words and emotional phrases in each category

\begin{tabular}{|l|c|c|}
\hline & Emo_words & Emo_phrases \\
\hline Expect & 2,141 & 399 \\
\hline Joy & 3,927 & 830 \\
\hline Love & 7,378 & 1,556 \\
\hline Surprise & 578 & 134 \\
\hline Anxiety & 4,817 & 1,074 \\
\hline Sorrow & 3,039 & 751 \\
\hline Anger & 1,509 & 318 \\
\hline Hate & 3,571 & 724 \\
\hline
\end{tabular}

\subsubsection{Emotional phrases}

Emotional phrases are composition of words so as to convey accurate emotions. We take the proverb as the example, generally, the proverb conveys the special emotional state.

E_phrase 1: “谢天谢地(thank goodness)”

This phrase represents the emotion of "grateful(joy)", while each single word could not express this emotion.

E_phrase 2: “善与恶(good and evil)”

This phrase includes two words, each word convey opposite sentiment. The phrase can emphasize one of sentiment when this phrase is used in the context.

In the Ren-CECps, many emotional phrases are given multiple emotion tags, so we use the same method as emotional words to determine the chief emotion of emotional phrases. The numbers of emotional phrases in each category are shown in Table 1.

\subsubsection{POS (part-of-speech) tags}

We are interested in the POS tags of all words, because words of some POS tags such as adjective and verb are more likely to convey sentiments [13], such as the adjective "pretty" may express emotion of love, and the verb "dislike" can express the feeling of hate.
Besides POS tags of adjective and verb, there are totally 41 kinds of POS tags in our data source.

\subsubsection{Degree words}

Degree words are always used for changing the intensity of emotion. They almost appear in the sentence which contains emotion in chinese textual, such as “很(very)”, “几乎(almost)” and “稍微(a little)”. There are two examples shown as follows:

D_word 1: (很)高兴(very) happy

D_word 2: (有点)遗憾(a little) regret

In the first example, the word “高兴(happy)" is modified by the degree word “很(very)", which always increases the emotion intensity of the emotion words or emotional phrases. In the second example, the word “遗憾(regret)” is modified by the degree word “有 点(a little)", which always decreases the emotion intensity of the emotional words or emotional phrases.

There are 1,039 different degree words annotated in Ren-CECps. The occurrences of degree words count 16,713 times, among which, 8,294 degree words have modified emotional words or emotional phrases.

\subsubsection{Negative words}

In Chinese articles, negative words, such as “不(no)”, “不会(cannot)” and “别(donot)”, appear with highfrequency. With negative words in sentences, meaning can be reversed while emotion types may be changed or not. The examples are shown as follows:

Sentence 1: “他希望下雪。(He hopes for snow.)”

Sentence 2: “他不希望下雪。(He doesn't hope for snow.)"

In comparison between two sentences above, we find that using negative word, only the meaning of sentence is changed. There are 645 different negative words annotated in Ren-CECps.

The negative words count for totally 13,750 times, among which, 3,668 negative words have modified emotional words or emotional phrases.

\subsubsection{Conjunctions}

People like using conjunctions in the complex sentences. On the one hand, conjunctions join simple sub-sentences into a long sentence. On the other hand, the occurrence of conjunctions may signify the change of emotion intensity. The examples are shown as follows:

Sentence 1: “为了升职, 他忽略了亲情和背叛 了朋友。(For promotion, he ignored the family and betrayed friends.)" 
Sentence 2: “为了升职, 他不但忽略了亲情而 且背叛了朋友。(For promotion, he not only ignored the family, but also betrayed friends.)"

Obviously, sentence 2 using conjunctions (not only ... but also) expresses stronger critical sentiment compared to sentence 1 .

There are 297 different conjunctions annotated in Ren-CECps. The total of conjunctions occur 12,900 times.

\subsection{Feature sets}

There are five feature sets in our experiment for emotional keywords annotation and emotion types recognition. Besides words and part-of-speech tags, we extract three modification features, namely, degree words, negative words and conjunctions.

As depicted in equation 3.1 to 3.5, we adopt n-gram models where n could be 1,2 or 3 for words $\left(F_{w}\right)$, corresponding POS tags $\left(F_{p o s}\right)$, and degree words modification $\left(F_{d m}\right)$, negative words modification $\left(F_{n m}\right)$ and conjunctions modification $\left(F_{c m}\right)$.

$$
\begin{aligned}
F_{w}= & \{U n i-\operatorname{gram}(\text { Word })\} \cup\{B i-\operatorname{gram}(\text { Word })\} \\
& \cup\{\text { Tri }-\operatorname{gram}(\text { Word })\}
\end{aligned}
$$

$$
\begin{aligned}
F_{p o s}= & \{U n i-\operatorname{gram}(P O S)\} \cup\{B i-\operatorname{gram}(P O S)\} \\
& \cup\{\operatorname{Tri}-\operatorname{gram}(P O S)\}
\end{aligned}
$$

$$
\begin{aligned}
F_{d m}= & \{1-\operatorname{gram}(D-\bmod )\} \cup\{2-\operatorname{gram}(D-\bmod )\} \\
& \cup\{3-\operatorname{gram}(D-\bmod )\}
\end{aligned}
$$

$$
\begin{aligned}
F_{n m}= & \{U n i-\operatorname{gram}(N-\bmod )\} \cup\{\operatorname{Bi}-\operatorname{gram}(N-\bmod )\} \\
& \cup\{\operatorname{Tr} i-\operatorname{gram}(N-\bmod )\}
\end{aligned}
$$

$$
\begin{aligned}
F_{n m}= & \{U n i-\operatorname{gram}(C-\bmod )\} \cup\{B i-\operatorname{gram}(C-\bmod )\} \\
& \cup\{\operatorname{Tr} i-\operatorname{gram}(C-\text { mod })\}
\end{aligned}
$$

where "D-mod", "N-mod", "C-mod" mean the modification relations between words and degree words, words and negative words, words and conjunctions respectively. In other words, D-mod means that the word(s) is(are) modified by the degree word, N-mod means that the word(s) is(are) modified by the negative word, and C-mod means that the word(s) is(are) modified by the conjunction.

In this study, we aim to annotate the emotional keywords and recognize the emotion types of the keywords. That is to say, our goal is to recognize the emotion state of each word, mark the keywords with corresponding emotion tags, while mark other words with No_Emo.

To illustrate these feature sets more specifically, we give a simple example as shown in Figure .1 for the short sub-sentence “虽然他不是很高兴(Though he is not very happy)". $Y 1, \ldots, Y 5$ denote the emotion states of the words in the sentence. There are five observation feature under each emotion state, which are word itself, part-of-speech, degree word modification, negative word modification and conjunction modification. The modification relations between words and these modifiers are represented by the dotted arrows.

Table 2 Tri-gram of emotion state $Y 4$

\begin{tabular}{|l|c|c|}
\hline & Emo_words & Emo_phrases \\
\hline Expect & 2,141 & 399 \\
\hline Joy & 3,927 & 830 \\
\hline Love & 7,378 & 1,556 \\
\hline Surprise & 578 & 134 \\
\hline Anxiety & 4,817 & 1,074 \\
\hline Sorrow & 3,039 & 751 \\
\hline Anger & 1,509 & 318 \\
\hline Hate & 3,571 & 724 \\
\hline
\end{tabular}

In this sentence, we have a conjunction “虽然(though)", a negative word “不是(isnot)" as well as a degree word “很(very)”. The words $\{$ 他(he), 不是(isnot), 很(very), 高兴(happy)\} are modified by the conjunction $\{$ 虽然(though) $\}$. The words $\{$ 很(very), 高兴(happy) $\}$ are modified by the negative word $\{$ 不是(isnot) . The word $\{$ 高兴 (happy) $\}$ is modified by the degree word $\{$ 很(very) $\}$.

Here, we enumerate tri-gram model of the above five feature sets for the state node $Y 4$ in Figure1 as shown in Table 2.

\subsection{Experiment}

In this study, we conduct eight experiments with different feature sets to explore whether modification features can improve the performance of emotional keywords annotation and emotion types recognition. As contextual information is under consideration, we think that the conditional random field would be the 
appropriate algorithm for capturing the relation between the words and their corresponding modifiers.

We focus on the effectiveness of these three modification features in the sentence level. We directly extract them and add them to the homologous feature set.

There are 35,096 sentences in the Ren-CECps corpus. But we find that there exist some sentences composed of punctuation or only an auxiliary word for pronunciation such as “啊(a)” and “吧(ba)", and these sentences could not contain any emotion keywords, thus we remove them from the datasource.

After we filtered out sentences which are composed by few words or punctuations, there are 31,070 sentences left for the experiment of emotional keywords annotation and emotion types recognition. We randomly select 24,856 sentences for training, and 6,214 sentences for testing.

\subsection{Experiment method}

(Lafferty et al., 2001) define the conditional random fields, which is the probability of label sequence $y$ given corresponding observation sequence $x$. The conditional distribution $p(Y \mid X)$ takes the form

$$
p(Y \mid X)=\frac{1}{Z(X)} \exp \left\{\sum_{k=1}^{k} \lambda_{k} f_{k}\left(y_{i}, Y_{i-1}, X_{i}\right)\right\}
$$

where $Z(X)$ is a normalizing factor; $\lambda k$ is a available parameter through training data; $f_{k}\left(y_{i}, y_{i-1}, X_{i}\right)$ is a set of feature functions, in which $X_{i}$ is the observation at position $i$ and $y$ are the labels at position $i$ and $i-1$ in label sequence.

\subsection{Feature selection}

We carried out eight experiments with different feature sets, which are listed in Table 3.

Because Ex_1 only used two features, which are words and part-of-speech, we regard the result of Ex_1 as the baseline.
Table 3 Feature sets for each experiment

\begin{tabular}{|l|c|c|c|c|c|}
\hline Experiments & \multicolumn{6}{|c|}{ Feature sets } \\
\hline & $F_{w}$ & $F_{p o s}$ & $F_{c m}$ & $F_{d m}$ & $F_{n m}$ \\
\hline$E x_{1}$ (baseline) & $\mathrm{o}$ & $\mathrm{o}$ & & & \\
\hline$E x_{2}$ & $\mathrm{o}$ & $\mathrm{o}$ & $\mathrm{o}$ & & \\
\hline$E x_{3}$ & $\mathrm{o}$ & $\mathrm{o}$ & & $\mathrm{o}$ & \\
\hline$E x_{4}$ & $\mathrm{o}$ & $\mathrm{o}$ & & & $\mathrm{o}$ \\
\hline$E x_{5}$ & $\mathrm{o}$ & $\mathrm{o}$ & $\mathrm{o}$ & $\mathrm{o}$ & \\
\hline$E x_{6}$ & $\mathrm{o}$ & $\mathrm{o}$ & $\mathrm{o}$ & & $\mathrm{o}$ \\
\hline$E x_{7}$ & $\mathrm{o}$ & $\mathrm{o}$ & & $\mathrm{o}$ & $\mathrm{o}$ \\
\hline$E x_{8}$ & $\mathrm{o}$ & & $\mathrm{o}$ & $\mathrm{o}$ & $\mathrm{o}$ \\
\hline
\end{tabular}

\subsection{Evaluation}

In evaluation part, precision, recall and F-score of emotional keywords for each emotion type are calculated using formula from 4.1 to 4.3 .

$$
\text { precision }=\frac{\text { num of correctly annotated emotions }}{\text { num of annotated emotions }}
$$

$$
\begin{aligned}
& \text { recall }=\frac{\text { num of correctly annotated emotions }}{\text { num of relevant emotions }} \\
& F-\text { score }=\frac{2 * \text { precision } * \text { recall }}{\text { precision }+ \text { recall }}
\end{aligned}
$$

\subsection{Results and discussion}

In the Figure 2, we can observe the average precision and recall of eight experiments adopted different feature sets. Ex_3 gets the best score in precision, while Ex_6 gets the worst one. In the recall, Ex_8 obtains the top value, while Ex_2 obtains the bottom value.

As shown in Figure.3, eight experiments get the different F-scores, among which Ex_8, Ex_7 and Ex_5

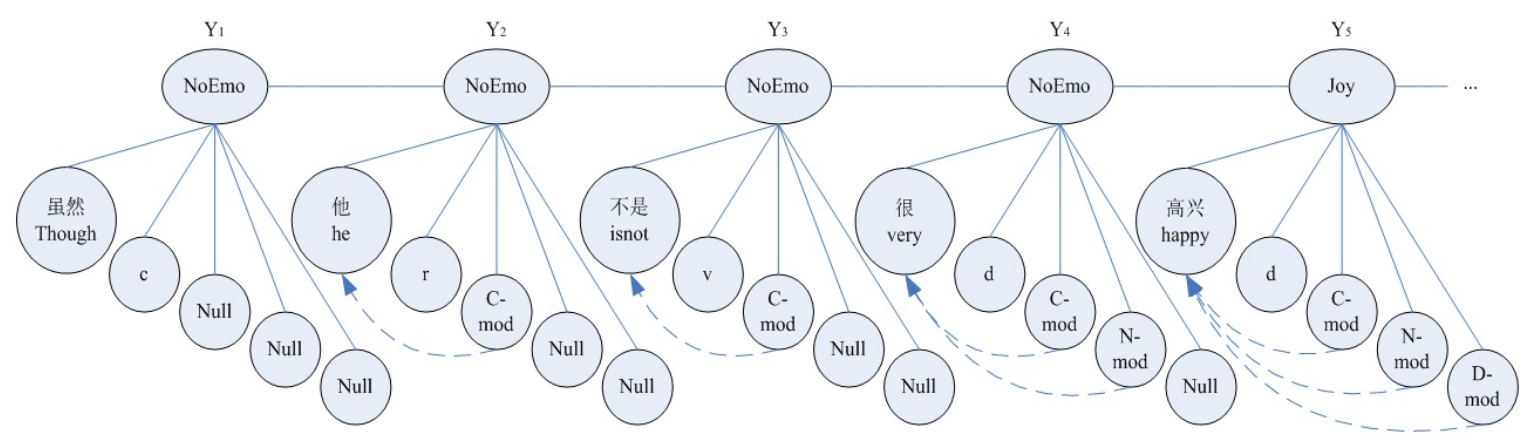

Figure 1 Recognition Errors 
get the best three F-scores, while Ex_3, Ex_6 and Ex_2 get the worst three F-scores.

When experiments just adopted one modification feature, such as Ex_2 and Ex_3, they get so bad results, even worse than the result of Ex_1 which can be seen as the baseline. Because with the degree words or conjunction in sentence, the emotion of keywords would arise variation in general, sometimes the polarity of keywords would transform into the inverse emotion state. So we can conclude that if we add conjunction or degree word into the basic feature sets (words and part-of-speech tags) directly, the result would go to worse. In other words, feature sets of \{words, part-ofspeech tags, conjunction \} and \{words, part-of-speech tags, degree words $\}$ are bad feature sets. Nevertheless, Ex_4 gets relatively medium F-score value, in which modification feature negative words have been added into the basic feature sets, meanwhile result of the Ex_4 is better than the baseline. In some way, it is proved that we can make use of negative words without any treatment as feature for improving the performance of emotional keywords annotation and emotion types recognition.

When we add two modification features into the basic feature sets, degree words and negative words would be the best choice as shown in the Figure 3 . The result of Ex_7 is better than the result of Ex_5 and Ex_6.

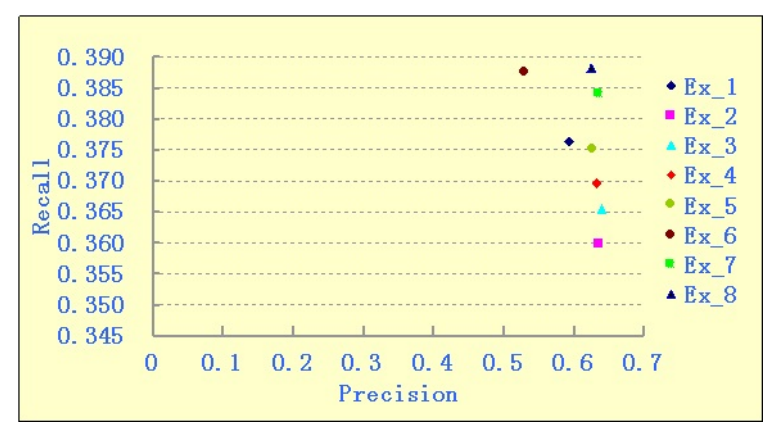

Figure 2 Recognition Errors

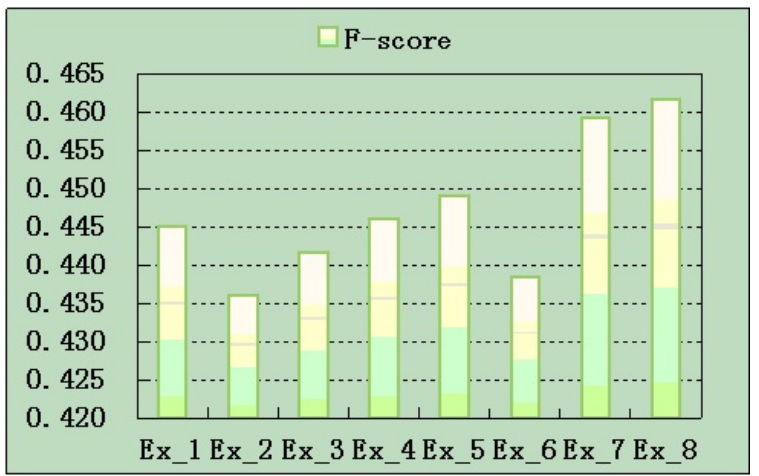

Figure 3 Recognition Errors
Ex_8, adopts the three modification features, gets the highest F-score, which proves the effectiveness of modification features for emotional keywords annotation and emotion types recognition.

For the further discussion, more detailed results of the eight emotion types in Ex_1 and Ex_8 are shown in Table 4 and Table 5 respectively.

Table 4 Precision, recall and F-score of Ex_1(baseline)

\begin{tabular}{|l|c|c|c|}
\hline Emotion & Precision & Recall & F-score \\
\hline No_emo & 0.939983 & 0.985561 & 0.962233 \\
\hline Joy & 0.577795 & 0.329599 & 0.419753 \\
\hline Hate & 0.500998 & 0.197482 & 0.283296 \\
\hline Love & 0.661024 & 0.535816 & 0.591871 \\
\hline Sorrow & 0.581892 & 0.350920 & 0.437811 \\
\hline Anxiety & 0.517260 & 0.355690 & 0.421523 \\
\hline Surprise & 0.489130 & 0.175781 & 0.258621 \\
\hline Anger & 0.482456 & 0.146277 & 0.224490 \\
\hline Expect & 0.589260 & 0.309924 & 0.406203 \\
\hline Av. & 0.593311 & 0.376339 & 0.445089 \\
\hline
\end{tabular}

Table 5 Precision, recall and F-score of Ex_8

\begin{tabular}{|l|c|c|c|}
\hline Emotion & Precision & Recall & F-score \\
\hline No_emo & 0.941841 & 0.987083 & 0.963931 \\
\hline Joy & 0.610493 & 0.330892 & 0.429170 \\
\hline Hate & 0.553704 & 0.235248 & 0.330204 \\
\hline Love & 0.673164 & 0.553813 & 0.607684 \\
\hline Sorrow & 0.586558 & 0.353374 & 0.441261 \\
\hline Anxiety & 0.531632 & 0.376790 & 0.441014 \\
\hline Surprise & 0.543210 & 0.171875 & 0.261128 \\
\hline Anger & 0.551724 & 0.170213 & 0.260163 \\
\hline Expect & 0.631902 & 0.314504 & 0.419980 \\
\hline Av. & 0.624914 & 0.388199 & 0.461615 \\
\hline
\end{tabular}

Compared Table 4 with Table 5, the precision, recall and F-score of Ex_8 are better than the result of Ex_1. Emotion of Love, Sorrow and Anxiety get the top three F-score with $59.19 \%, 43.78 \%, 42.15 \%$ in Ex_1 and with $60.77 \%, 44.13 \%, 44.10 \%$ in Ex_8, while emotion of Surprise, Hate and Anger get the bottom three with $25.86 \%, 28.33 \%, 22.45 \%$ in Ex_1 and with $26.11 \%, 33.02 \%, 26.01 \%$ in EX_8. So we are able to come to a conclusion that there is a pretty clear distinction between emotions of Love, Sorrow and Anxiety, while distinguishing the Surprise, Hate and Anger emotions is found to be difficult.

The distinction between Hate and Anger turns to be much difficult to make. For one reason, a great part of 
emotional words and phrases convey two or more passive emotions at the same time, which makes it impossible even for human to annotate. Take the following sentence as an example,

Sentence : “我无法忍受他对我的欺骗。( I can't stand him cheating on me. )"

One corpus annotator labeled the word “欺骗 (cheating)" with emotion of Anger, while others labeled with Hate or both of them. For the emotion of Surprise, we believe that the lack of training data leads to the low recall rate directly.

As shown in Table 1, the number of keywords conveying the Surprise emotion turns to be the minimum. Compared with the top three emotions with highest Fscores, the number of words with Surprise annotated counts only $7.97 \%$ of the words with Love annotated, and $18.79 \%$ of Sorrow annotated, and $12.09 \%$ of Anxiety.

\section{Conclusion}

In this study, we have carried out eight experiments with different feature sets on emotional keywords annotation and emotion types recognition. We capture the relevance between emotion keywords and modification feature, using Conditional Random fields, in order to explore the effectiveness of the modification relations. The experiment results reveal Ex_8 outperforms other experiments, which proves that modification features are effective for improving the performance of emotional keywords annotation and emotion types recognition. Meanwhile, we conclude that when we only adopt one modification feature without any pre-process for the experiment, the negative words would be the best choice, while adopting conjunctions or degree words would cause the bad result. And if we adopt two modification feature sets, employing degree words and negative words as features can optimize the result. For our future work, we will look for better methods for distinguishing the similar emotions such as "Hate" and "Anger". And we also think about how to treat the conjunctions for optimizing the performance of emotional keywords annotation and emotion types recognition.

\section{Acknowledgment}

This work was supported in part by a grant from the Grant-in-Aid for Scientific Research (B) numbered 21300036 and Young Scientists (B) numbered 23700252 from the Japan Society for the Promotion of Science, and the Ministry of Education, Science, Sports and
Culture, Grant-in-Aid for Challenging Exploratory Research, 21650030.

\section{References}

[1] M. Hu and B. Liu, "Mining and Summarizing Customer Reviews", Proceedings of the ACM SIGKDD Conference on Knowledge Discovery and Data Mining (KDD), pp.168-177, 2004.

[2] R. Tokuhisa, K. Inui, and Y. Matsumoto (Eds.), "Emotion Classification Using Massive Examples Extracted from the Web", Proceedings of COLING, pp.881-888, 2008.

[3] Xin Kang, Fuji Ren and Yunong Wu, "Bottom Up: Exploring Word Emotions for Chinese Sentence Chief Sentiment Classification", Proceedings of 6th International Conference on Natural Language Processing and Knowledge Engineering, 2010.

[4] Changqin Quan and Fuji Ren, "Construction of a Blog Emotion Corpus for Chinese Emotional Expression Analysis", Proceedings of the 47th Annual Meeting of the Association for Computational Linguistics, 2009.

[5] Vincent Ng and Sajib Dasgupta and S. M. Niaz Arifin, "Examining the role of linguistic knowledge sources in the automatic identification and classification of reviews", Proceedings of the COLING/ACL, 2006.

[6] J. Lafferty, A. McCallum, and F. Pereira, "Conditional random felds: Probabilistic models for segmenting and labeling sequence data", Proceedings of International Conference on Machine Learning-01, 2001.

[7] Bo Pang and Lillian Lee and Shivakumar Vaithyanathan, "Thumbs up? Sentiment Classification using Machine Learning", Proceedings of the Conference on Conference on Empirical Methods in Natural Language Processing, July 2002.

[8] Peter D. Turney, "Thumbs Up or Thumbs Down? Semantic Orientation Applied to Unsupervised Classification of Reviews", Proceedings of the 40th Annual Meeting of the Association for Computational Linguistics, Philadelphia, July 2002.

[9] Michael Gamon, "Sentiment classification on customer feedback data: noisy data, large feature vectors, and the role of linguistic analysis", In Proceedings the 20th International Conference on Computational Linguistics, 2004.

[10] Changhua Yang, Kevin Hsin-Yih Lin, Hsin-Hsi Chen, "Building Emotion Lexicon from Weblog Corpora", Proceedings of the 45th Annual Meeting of the Association for Computational Linguistics, 2007.

[11] Yunong Wu, Kenji Kita, Fuji Ren, Kazuyuki Matsumoto and Xin Kang, "Modification Relations 
based Emotional Keywords Annotation using Conditional Random Fields", Proceedings of the 4th International Conference on Intelligent Networks and Intelligent Systems, November 2011, Kunming, China.

[12] Shotaro Matsumoto, Hiroya Takamura, and Manabu Okumura, "Sentiment Classification Using Word Sub-sequences and Dependency Sub-trees", Proceedings of the 9th Pacific-Asia Conference on Knowledge Discovery and Data Mining, 2005.

[13] P. Chesley, B. Vincent, L. Xu, and R. Srihari, "Using verbs and adjectives to automatically classify blog sentiment,", AAAI Symposium on Computational Approaches to Analysing Weblogs (AAAI$C A A W)$, pp.27-29, 2006.

[14] Jun Zhao, Kang Liu, Gen Wang. "Adding Redundant Features for CRFs-based Sentence Sentiment Classification", Proceedings of Empirical Methods in Natural Language Processing, 2008.

[15] Yang, K. H.-Y. Lin, and H.-H. Chen, "Emotion Classification from Web Blog Corpora", IEEE/WIC/ACM, 2007.

[16] Dipankar Das and Sivaji Bandyopadhyay, "Word to Sentence Level Emotion Tagging for Bengali Blogs", Proceedings of the ACL-IJCNLP 2009 Conference Short Papers, pp.149-152, Suntec, Singapore, 2009.

[17] Ortony, A., G. L. Clore, and M. A. Foss, "The psychological foundations of the affective lexicon”, Journal of Personality and Social Psychology, 53:751-766, 1987.
[18] J. M. Wiebe and E. Riloff, "Creating subjective and objective sentence classifiers from unannotated texts," in Proceedings of the Conference on Computational Linguistics and Intelligent Text Processing (CICLing), number 3406 in Lecture Notes in Computer Science, pp.486-497, 2005.

[19] Antonio Reyes and Paolo Rosso,"Mining Subjective Knowledge from Customer Reviews:A Specific Case of Irony Detection", Proceedings of the 2nd Workshop on Computational Approaches to Subjectivity and Sentiment Analysis, ACL-HLT 2011, pp.44-52, Portland, Oregon, USA, 24 June, 2011.

[20] Adnan Duric and Fei Song, "Feature Selection for Sentiment Analysis Based on Content and Syntax Models", Proceedings of the 2nd Workshop on Computational Approaches to Subjectivity and Sentiment Analysis, ACL-HLT 2011, pp.44-52, Portland, Oregon, USA, 24 June, 2011.

[21] Fuji Ren, "Affective Information Processing and Recognizing Human Emotion", Electronic Notes in Theoretical Computer Science, Vol.225, No.2009, pp.39-50, 2009. 